\title{
Experimental Study of the Sediment Trap Effect of Steel Grid-Type Sabo Dams
}

\author{
Teruyoshi TAKAHARA ${ }^{1}$ and Kazuki MATSUMURA ${ }^{1}$ \\ ${ }^{1}$ Graduate school of Agriculture, Kyoto Pref. University (1-5 Shimogamonakaragicyo, Sakyo-ku, Kyoto, Kyoto 6068522, Japan)
}

\begin{abstract}
Landslides caused by rain often take the form of earth flows when they reach their final stage in river valleys. Conventional steel grid- or slit-type sabo dams were originally developed for debris flows containing boulders at their leading edge and do not contain earth flows as effectively as they contain the flows for which they were designed. If the volume behind these types of dams is not quickly filled by a debris flow, subsequent earth flows pass through the dam, because these subsequent flows are composed of less coarse rock and gravel. Therefore, we designed and built several model sabo dams with closely-spaced horizontal units. We then conducted hydraulic experiments focusing on the effects of these closely-spaced horizontal units, with the objective of ensuring that they capture materials in the later phases of debris flows. Experimental results indicated that this new design of sabo dam is better at capturing finer sediments in both debris flows and earth flows.
\end{abstract}

\section{INTRODUCTION}

Steel grid-type sabo dams are considered to have a minimal impact on riverine ecology because, under ordinary conditions, they do not block the river course. For this reason, recent research has focused on this type of dam. The grids are constructed of steel pipes set in a lattice pattern. However, the larger the grid is, the more skill is required for its assembly; this one of the primary restrictions associated with these dams. Consequently, authorities are interested in other sabo dam designs that may be equally effective at trapping sediment but require fewer pipe units. Takahara et al. (2007) described the characteristics of steel pipe slit-type dams that use vertical or horizontal units to trap earth flows. They found that it is possible to develop sabo dams that are capable of efficiently trapping sediment on stream beds with a gentle slope, where sediment transport takes the form of earth flows, using lattices composed of vertical and horizontal units of differing diameters.

According to Ishikawa [1985] and Suwa [1988], debris flows are characterized by an initial concentration of the largest components of rubble, followed by finer sediments. However, once the dam becomes filled with trapped sediments, finer sediments can be carried away by subsequent flooding events. Any large sediment flow through a dam will thus have an effect downstream.

Numerous studies have been conducted on steel grid-type sabo dams [e.g., Ashida et al. 1980,1987; Mizuno et al. 1996, 1987; Mizuyama et al. 1995; Takahashi et al. 2001a, 2001b; Watanabe et al. 1980], but none appears to have assessed the downstream effects that occur when these dams fail to trap sediment. The purpose of this study was to develop a dam that is highly efficient at trapping sediment when it is situated on a gentle gradient, and to test various versions of the design using hydraulic experiments.

\section{MATERIALS AND METHODS}

The experiments used a scale of 1:40. Sand (particle size: $0.84 \mathrm{~mm}$ ) was bonded to the channel bottom. Figure 1 shows a diagram of the channel and Figure 2 shows the gradation of the sediment used in the experiment.

\subsection{New dam concept}

The experiment focused on outflows of trapped sediments following debris flows. We employed a new design of sabo dam with the goal of blocking such outflows. Figure 3 shows a conventional sabo dam (Type 1) and Figure 4 shows the experimental design (Type 2). The experimental dam differs from the conventional dam in that it has thin, closely-spaced, horizontal units attached to the downstream side of the dam. The spacing from the bottom horizontal unit to the ground was adjustable, as shown in Figure 4 (c). Three Type 2 configurations (Types 2-1, 2-2, and 2-3) were 
constructed and tested; the horizontal units were spaced $0.5,1.0$, and 2.0 times the $\mathrm{D}_{95}$ of the experimental sand, respectively. Figure 5 shows a Type $3 \mathrm{dam}$; in this dam, horizontal units had identical diameters to those in Type 2, but they were located on the upstream side of the dam. Figure 6 shows an upstream inclined grid-type model dam, with the horizontal units and vertical units equally spaced. Figure 7 shows an upstream inclined slit-type dam with horizontal beams spaced 3.0 times the $\mathrm{D}_{95}$ of the experimental sand, a $3.0 \times 1.5$ grid-type dam. The models shown in Figures 1, 5, 6, and $\mathbf{7}$ were constructed and tested for comparison with the experimental designs (Figure 4).

\subsection{Experimental conditions}

Table 1 shows the experimental conditions and cases used in this study. The channel slope $(\theta)$ was set at $16^{\circ}$ (Conditions I and III ) and $12^{\circ}$ (Conditions II and IV). Water discharge (Q) was set at $2.5 \mathrm{1} / \mathrm{s}$ (Conditions I and III) and $3.0 \mathrm{l} / \mathrm{s}$ (Conditions II and IV ). Conditions I and III assumed a debris flow, while Conditions II and IV assumed an earth flow. " $T_{1}$ seconds" indicates the period of time during which sediment (in either a debris flow or an earth flow) is trapped behind the sabo dam, ending when subsequent flow from the upstream tip of the accumulated sediment has stopped and no further washing occurs. $T_{2}$ was a

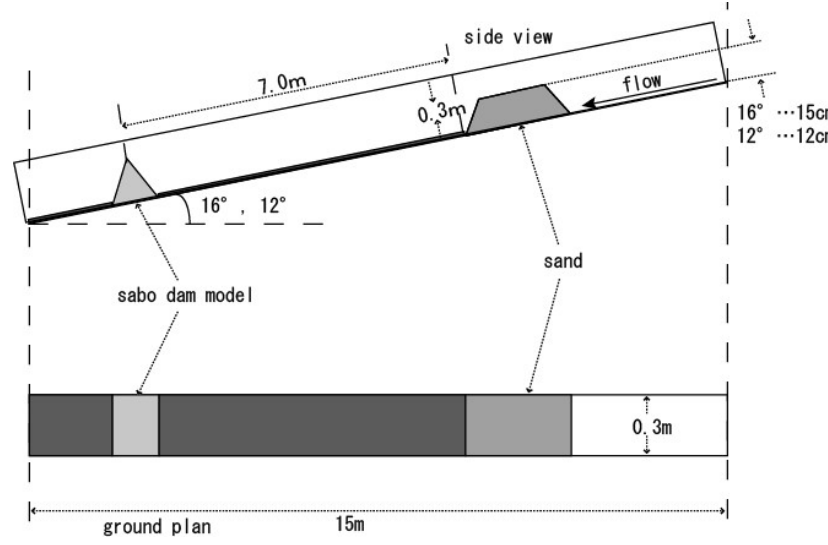

Fig.1 Experiment channel

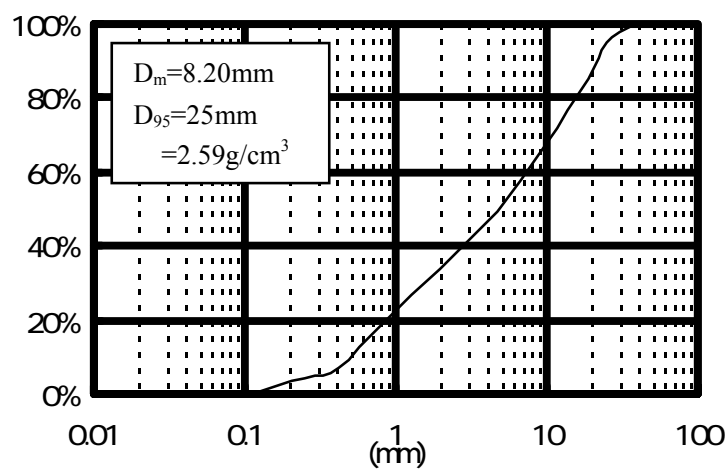

Fig.2 Grain size distribution of the using materials fixed time period set for subsequent flows. $\mathrm{Q}, \mathrm{T}_{1}$, and $\mathrm{T}_{2}$ were set based on data obtained from a preliminary experiment. Conditions I and II assumed that the reservoir was filled with sediment from the river flow during time $T_{1}$. Conditions III and IV assumed that the river flow was not sufficient to fill the reservoir with sediment during $\mathrm{T}_{1}$.

All model dams were tested under Conditions I and III. All dams were also tested under

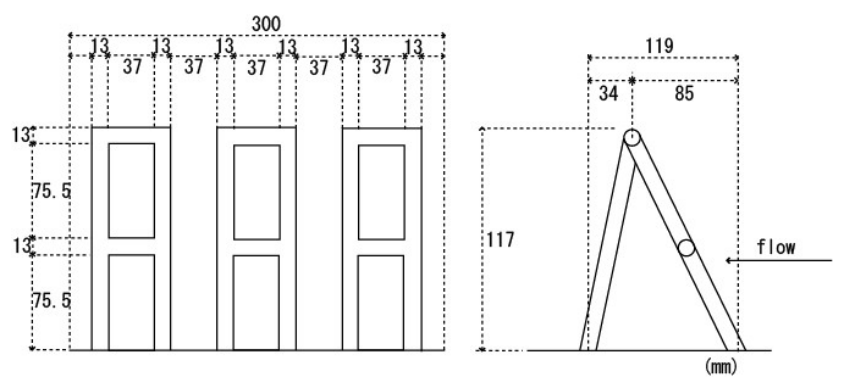

Fig.3 type-1 model

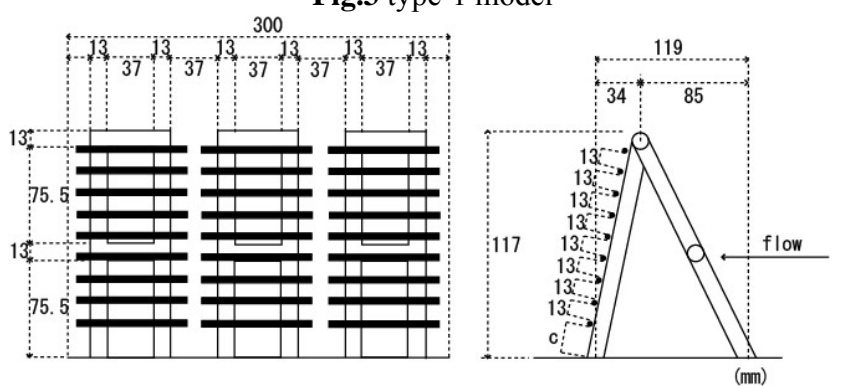

Fig.4 type-2 model

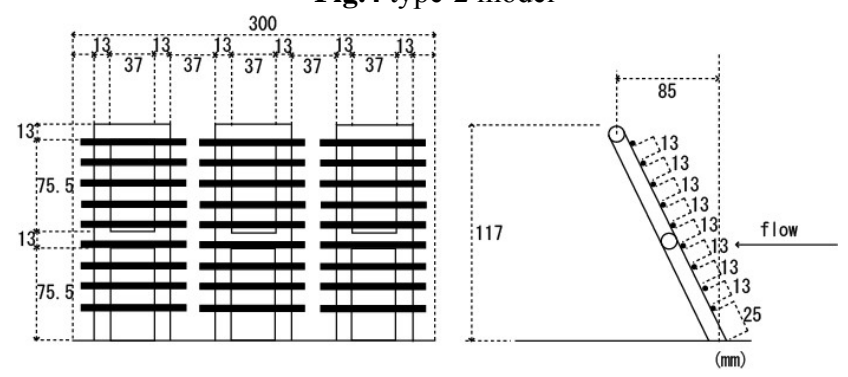

Fig.5 type-3 model

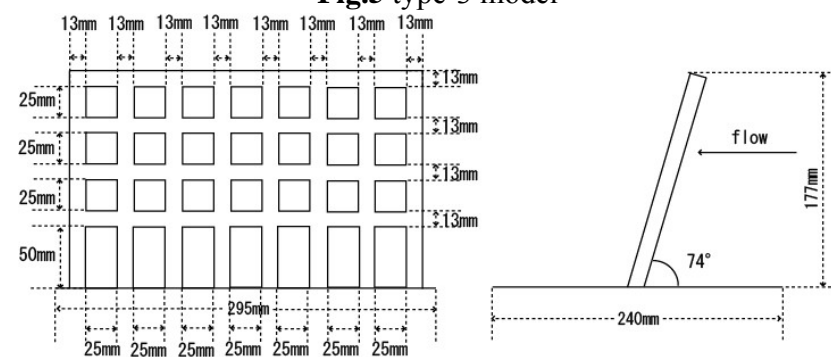

Fig.6 grid-type model

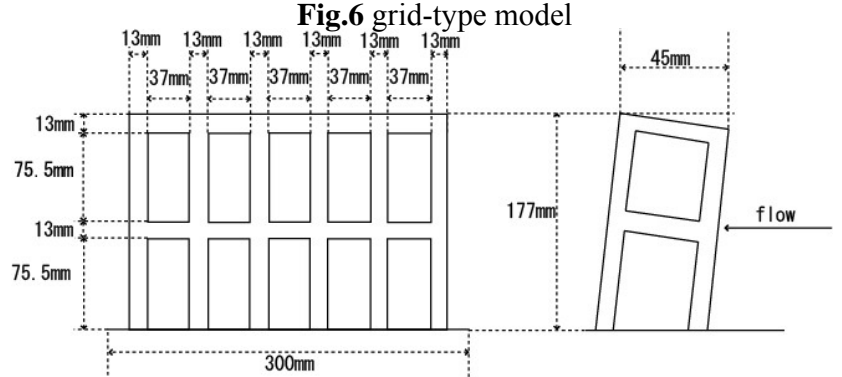

Fig.7 grid 3.0×1.5 type model 
Table1 Experimental cases

\begin{tabular}{|c|c|c|c|c|c|c|c|c|}
\hline \\
\hline Run-No. & condition & dam model & $\theta\left({ }^{\circ}\right)$ & $\mathrm{Q}(\ell / \mathrm{s})$ & $\mathrm{V}(\ell)$ & $\mathrm{T}_{1}(\mathrm{~s})$ & $\mathrm{T}_{2}(\mathrm{~s})$ & mode \\
\hline 1 & \multirow{7}{*}{1} & type-1 & \multirow{7}{*}{16} & \multirow{7}{*}{2.5} & \multirow{7}{*}{32.2} & \multirow{7}{*}{7} & \multirow{7}{*}{20} & \multirow{7}{*}{$\begin{array}{l}\text { debris } \\
\text { flow }\end{array}$} \\
\hline 2 & & type- $2-1$ & & & & & & \\
\hline 3 & & type-2 - 2 & & & & & & \\
\hline 4 & & type- $2-3$ & & & & & & \\
\hline 5 & & type-3 & & & & & & \\
\hline 6 & & grid & & & & & & \\
\hline 7 & & grid-3.0×1.5 & & & & & & \\
\hline 8 & \multirow{5}{*}{ II } & type-1 & \multirow{5}{*}{12} & \multirow{5}{*}{3.0} & \multirow{5}{*}{47.2} & \multirow{5}{*}{25} & \multirow{5}{*}{20} & \multirow{5}{*}{$\begin{array}{l}\text { earth } \\
\text { flow }\end{array}$} \\
\hline 9 & & type- $2-1$ & & & & & & \\
\hline 10 & & type-2 - 2 & & & & & & \\
\hline 11 & & type-2 - 3 & & & & & & \\
\hline 12 & & grid-3.0×1.5 & & & & & & \\
\hline 13 & \multirow{7}{*}{ III } & type-1 & \multirow{7}{*}{16} & \multirow{7}{*}{2.5} & \multirow{7}{*}{16.1} & \multirow{7}{*}{2} & \multirow{7}{*}{15} & \multirow{7}{*}{$\begin{array}{l}\text { debris } \\
\text { flow }\end{array}$} \\
\hline 14 & & type- $2-1$ & & & & & & \\
\hline 15 & & type-2 - 2 & & & & & & \\
\hline 16 & & type-2 - 3 & & & & & & \\
\hline 17 & & type-3 & & & & & & \\
\hline 18 & & grid & & & & & & \\
\hline 19 & & grid-3.0×1.5 & & & & & & \\
\hline 20 & \multirow{5}{*}{ IV } & type-1 & \multirow{5}{*}{12} & \multirow{5}{*}{3.0} & \multirow{5}{*}{23.6} & \multirow{5}{*}{10} & \multirow{5}{*}{15} & \multirow{5}{*}{$\begin{array}{l}\text { earth } \\
\text { flow }\end{array}$} \\
\hline 21 & & type-2 - 1 & & & & & & \\
\hline 22 & & type- $2-2$ & & & & & & \\
\hline 23 & & type- $2-3$ & & & & & & \\
\hline 24 & & grid-3. $0 \times 1.5$ & & & & & & \\
\hline
\end{tabular}

Conditions II and IV with the exception of Type 3 and grid-type dams; these were not included because they have closely-spaced units and should be able to trap a large quantity of sediment when the channel gradient is $12^{\circ}$ (Conditions II and IV).

\subsection{Measured parameters}

We estimated the sediment trap rate $(\%), f_{l}$, and outflow rate $(\%), f_{2}$, and assessed the performance of the sabo dam design based on these two parameters. Equations (1) and (2) were used to calculate $f_{1}$ and $f_{2}$. $\mathrm{V}$ was the volume of sediment used in the experiment, $V_{l}$ was the volume trapped behind the dam model during time period $\mathrm{T}_{1}$, and $V_{2}$ was the runoff volume during the time period at $\mathrm{T}_{2}$. Figure 8 provides diagrams for $V_{1}$ and $V_{2}$.

$$
\begin{aligned}
& f_{1}=\left(V_{1}-V_{2}\right) / V \times 100(\%) \\
& f_{2}=V_{2} / V \times 100(\%)
\end{aligned}
$$

\section{EXPERIMENTAL RESULTS AND OBSERVATIONS}

Figure 9 illustrates the experimental results.

\subsection{Channel gradient of $16^{\circ}$ and sediment trap conditions}

Under Condition I, the dam models did not differ greatly in sediment trap rate $\left(f_{l}\right)$; see Fig. 9-I. In contrast, under Condition III (Fig. 9-III), which assumed that the reservoir would be less than completely full of sand after $T_{1}, f_{l}$ values were lower for the Type 1 and $3.0 \times 1.5$ grid-type dams. 


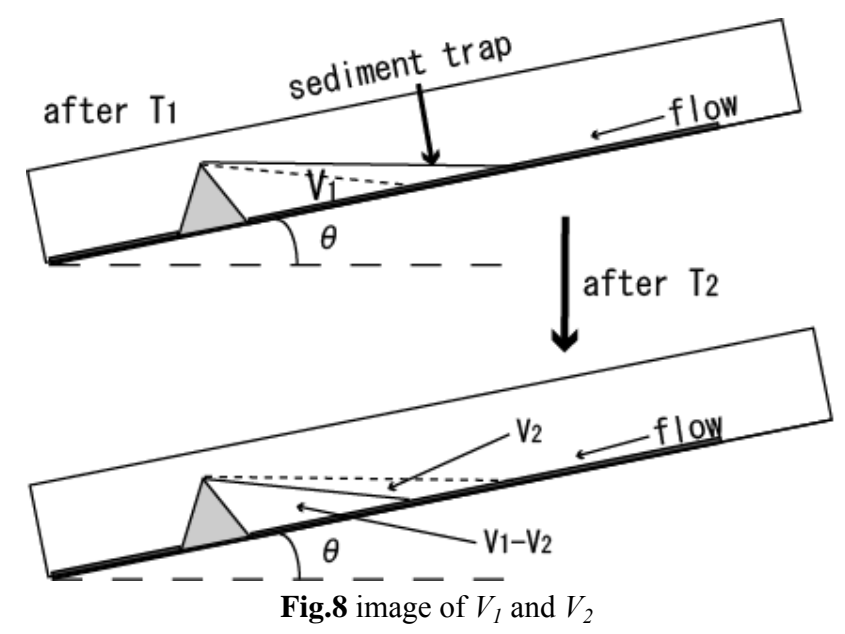

We observed a grain-size sorting effect in the sediment downstream of the dam in Condition I with a $16^{\circ}$ gradient. Sorting was characterized by the presence of boulders at the leading edge of the flow mass. It resulted in sediment's being trapped by all grids upstream of the dams and in completely full sand reservoirs. This finding explains why the models did not differ greatly in the volume of sediment washed downstream during subsequent flows. Photo 1 illustrates the sediment capture; no sediment appeared in dam reservoirs, but only behind the grid-type sabo dams upstream. Conversely, in Condition III, large quantities of gravel and finer materials were washed downstream during $\mathrm{T}_{2}$. Type 1 and $3.0 \times 1.5$ grid-type sabo dams, which lacked closely-spaced horizontal units, were unable to trap this sediment. Therefore, $f_{2}$ values were higher for Type 1 and $3.0 \times 1.5$ grid-type sabo dams than for other types of sabo dams.

Thus, additional horizontal units appear to be unnecessary in sabo dams located in steep riverbeds (gradients of $16^{\circ}$ or more), because their reservoirs can be expected to fill completely after a single sediment flow.

\subsection{Sediment trapping on a $12^{\circ}$ gradient}

We did not observe any grain-size sorting under experimental Conditions II (Fig. 9-II) or IV (Fig. 9-IV). Sediment flowed through the upstream steel grid-type sabo dams without being trapped. Downstream, the Type 2 sabo dam exhibited a high sediment trap rate. Photo 2 illustrates a typical case of trapping, clearly showing the larger particles that entered the dam and were trapped by the horizontal reinforcing units. This result was due to the characteristic pattern of earth flows: particles (corresponding to "boulders" and "cobbles" in this model) were not gathered at the leading edge of the moving sediment mass. This design exhibited a high trap ratio under this condition and under Condition IV, which also assumed only a half-full volume trapped by the dam.
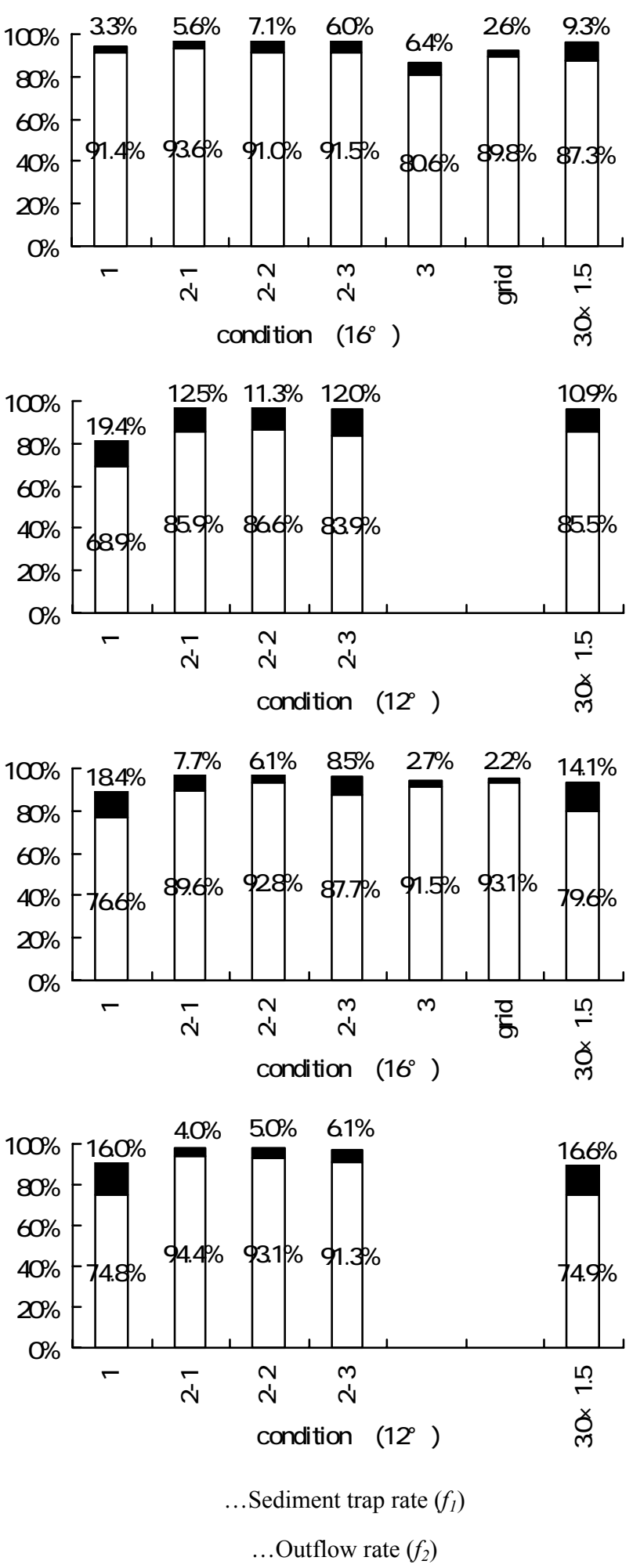

Fig.9 Experimental results

\subsection{Sediment trapping and grid spacing}

We tested three versions of the Type 2 sabo dam; trap rates did not differ under any experimental conditions. Interestingly, rates did not differ even on a $12^{\circ}$ gradient, where sand entered the reservoir as an earth flow.

Under such conditions, Type 2-3, which had the 
widest spacing, yielded the greatest advantages in terms of its influence on the adjacent environment.

\subsection{Utility of new dam types on streams with gentle gradients}

In terms of capacity and the construction process, it is more efficient to construct sabo dams on gentle slopes than on steep slopes. Our results indicated that conventional steel grid-type sabo dams on gentle slopes are relatively efficient at trapping granular flows. In addition, steel slit-type dams on gentle slopes (Conditions II and IV) are often able to trap driftwood due to their closely-spaced horizontal units. Sediment is subsequently captured behind the driftwood. If a flow contains no driftwood to be trapped, sediment is likely to pass through the dams, as shown in several of the cases tested in this study.

The Type 2 model, which incorporates closely-spaced horizontal reinforcing beams, exhibited high trapping efficiency even on gentle slopes where size-sorting processes do not occur. This design can be used in situations where earth flows are expected.

The high sediment trap rates $\left(f_{1}\right)$ of the Type 3 and grid-type dams provide further evidence that the Type 2 designs are likely to be relatively effective for trapping sediment on riverbeds with gentle slopes.

\section{CONCLUSIONS}

We obtained the following findings:

1. A granular flow in a riverbed with a gentle slope takes the form of an earth flow. Therefore, steel gridor slit-type sabo dams, which are designed to allow the passage of certain materials, are not as functionally stable as currently believed, because they are designed to take advantage of the natural size-sorting processes in a debris flow.

2. The proposed dam design, incorporating thin horizontal units, shows considerable potential for trapping sediment in both primary and subsequent flows on gentle slopes, which do not necessarily exhibit the size-sorting process associated with sediment retention.

ACKNOWLEDGEMENTS: The authors are grateful to JFE Kenzai for supporting this project.

\section{REFERENCES}

Ashida, K. and Takahashi, T. (1980): Study of debris flow control: Hydraulic function of grid type open dam, Annuals. Disaster Prevention Res. Inst., Kyoto Univ., No. 23B-2, pp. 433-441 (in Japanese with English synopsis).

Ashida, K. Egashira, S. Kurita, M and Araki, H. (1987): Debris

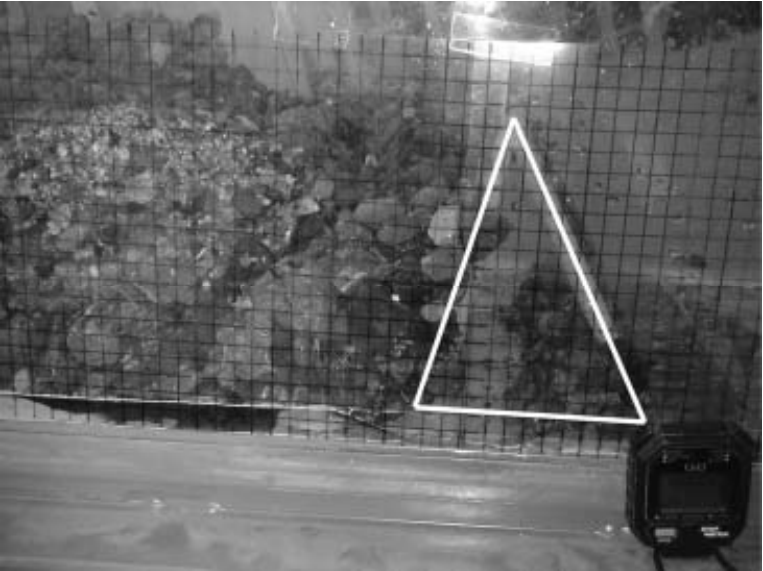

Photo.1 Experimental result (condition I type2)

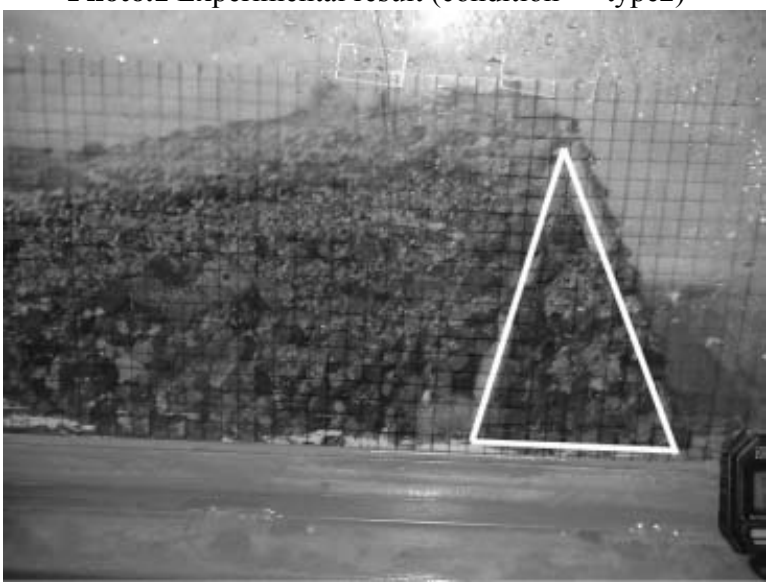

Photo.2 Experimental result (condition IV type2)

flow control by grid dams, Annuals. Disaster Prevention Res. Inst., Kyoto Univ., No. 30B-2, pp. 441-456 (in Japanese with English synopsis).

Ishikawa, Y. (1985): Debris flows in the Name River, Journal of the Japan Society of Erosion Control Engineering, Vol. 37, No. 5, pp. 24-29 (in Japanese).

Mizuno, H. and Mizuyama, T. (1996): Experimental study of a grid dam with narrow pipe interval of upper grids, Journal of the Japan Society of Erosion Control Engineering, Vol. 49, No. 4, pp. 3-8 (in Japanese with English abstract).

Mizuno, H. Minami, N. and Mizuyama, T. (2000): Experimental study of controlling debris flow by a consecutive series of open-type steel dams, Journal of the Japan Society of Erosion Control Engineering, Vol. 53, No. 1, pp. 19-25 (in Japanese with English abstract).

Mizuyama, T. Kobashi, S. and Mizuno, H. (1995): Control of passhing sediment with grid-type dams, Journal of the Japan Society of Erosion Control Engineering, Vol. 47, No. 5, pp. 8-13 (in Japanese with English abstract).

Suwa, H. (1988): Focusing mechanism of large boulders to a debris-flow front, Annuals. Disaster Prevention Res. Inst., Kyoto Univ., No. 31B-1, pp. 139-151 (in Japanese with English abstract).

Takahara, T. and Matsumura, K. (2007): Evaluation of sediment trap of ability based on sediment made and members distance, Journal of the Japan Society of Erosion Control 
Engineering, Vol. 60, No. 4, pp. 55-60 (in Japanese with English abstract).

Takahashi, T. Nakagawa, H. Satofuka, Y. and Wang, H. (2001): Simulation of debris flow deposition by open-type sabo dam, Annuals. Disaster Prevention Res. Inst., Kyoto Univ., No. 49B-2, pp. 133-139 (in Japanese with English synopsis).

Takahashi, T. Nakagawa, H. Satofuka, Y. and Wang, H. (2001):
Stochastic model of blocking for a grid-type dam by large boulders in debris flow, Journal of Hydroscience and Hydraulic Engineering, Vol. 45, pp. 79-84 (in Japanese with English abstract).

Watanabe, M. Mizuyama, T. and Uehara, S. (1980):

Deliberation about structure against debris flow, Journal of the Japan Society of Erosion Control Engineering, Vol. 32, No. 4, pp. 40-45 (in Japanese). 